\title{
Photometric and spectroscopic study of V 1184 Tauri
}

\author{
E. H. Semkov ${ }^{\star}$ \\ Institute of Astronomy, Bulgarian Academy of Sciences, and Isaac Newton Institute of Chile, Bulgarian Branch, 72, \\ Tsarigrdsko Shose blvd., 1784 Sofia, Bulgaria \\ Received 4 November 2002 / Accepted 21 March 2003

\begin{abstract}
We present results from optical photometric and spectroscopic observations of the pre-main sequence star V 1184 Tau. The star is associated with the Bok globule CB 34 and it was considered as a FUOR candidate in previous studies. Our CCD photometric data (UBVRI) obtained from October 2000-April 2002 show non-periodic changes in the brightness with amplitude of about $0.6(\mathrm{~V})$. In the period of observations, V 1184 Tau remained at the maximum brightness without increasing or decreasing. Two medium dispersion optical spectra of V 1184 Tau were obtained in 2001. Significant changes in the profile and strength of the emission peak in the $\mathrm{H} \alpha$ line were found. The [OI] line ( $\lambda$ 6003) is also seen in emission while the sodium doublet is still in a deep absorption. The comparison of our photometric and spectroscopic results suggests that V 1184 Tau has the characteristics of a WTTS
\end{abstract}

Key words. stars: pre-main sequence - stars: individual: V 1184 Tau

\section{Introduction}

$\mathrm{T}$ Tauri stars (TTSs) are a widespread class of pre-main sequence objects known from the pioneering work of Joy (1945). They are determined as young low-mass stars $\left(M \leq 2 M_{\odot}\right)$ with emission spectra and irregular photometric variability. The classical definition of TTSs given by Herbig (1962) was updated by Basian et al. (1983) and by Martin (1997). It is generally accepted that TTSs can be separated into two subclasses: Classical T Tauri stars (CTTSs) surrounded by an extended circumstellar disk and Weak line T Tauri stars (WTTSs) without evidence of disk accretion (Appenzeller \& Mundt 1989; Bertout 1989). According to Herbst et al. (1994), photometric variability of WTTSs is mainly produced by the presence of large photospheric cool spots analogous to sunspots. The periods of variability on time scales of days and amplitudes up to $0.8 \mathrm{mag}$ in $V$ are observed in WTTSs. Variability of CTTSs is more complicated: the presence of cool and hot surface spots produces non-periodic variations with amplitudes up to $3 \mathrm{mag}$ in $V$.

A very rare phenomenon in pre-main sequence evolution is the FU Orionis (FUOR) outburst (Herbig 1977). An increase in optical brightness of the order of 4-5 mag, a F-G supergiant spectrum with broad blue-shifted Balmer lines, strong infrared excess and connection with reflection nebulae are the main characteristics of FUORs. The observations in the case of the FUOR outburst is explained by increasing accretion caused by thermal instabilities in the circumstellar disk (Hartmann \& Kenyon 1996). From the ten objects classified as FUORs only

\footnotetext{
* e-mail: evgeni@skyarchive.org
}

three (FU Ori, V 1057 Cyg and V 1515 Cyg) have detailed photometric observations taken during the outburst.

It is now generally accepted that stars form in groups called stellar associations or stellar aggregates. While the highmass star formation takes place generally in the big molecular clouds, the low-mass stars can be formed even in the smallest regions such as Bok globules. They are small dark clouds of cold gas and dust where groups of a few Young Stellar Objects (YSO) can be observed.

The first evidence for star formation in the Bok globule CB34 (Clemens \& Barvainis 1988) was reported by Alves \& Yun (1995). They detected 12 objects connected with this globule that were found to exhibit near infrared color excesses typical of YSOs. Recently Khanzadyan et al. (2002) presented new evidence for star formation in CB 34. Two long well collimated $\mathrm{H}_{2}$ jets extending to the edge of the dark cloud were discovered. The authors suggest two phases of star formation in this region over time scales of few $\times 10^{5} \mathrm{yr}$.

Alves (1995) discovered the first variable object in this region named CB34V (or CB34FU). Comparison of CCD frames obtained in 1993 with the Palomar Observatory plates reveals the increasing brightness of this object of $3.7 \mathrm{mag}$ in the red. The first photometric and spectroscopic observations of this object were presented by Yun et al. (1997). The authors suspect a FUOR nature for this object on the basis of: the observed outburst in optical wavelengths, the presence of the object in the region of star formation, connection with a diffuse nebula and observed infrared excess. On the intermediate resolution spectrum to be presented here only the sodium doublet was seen in absorption. The authors suspect the presence of a $\mathrm{Li}(\lambda 6707)$ absorption line, but no evidence for an emission or absorption 
Table 1. CCD cameras and chip specifications.

\begin{tabular}{llll}
\hline \hline Telescope & $2-\mathrm{m} \mathrm{RCC}$ & $1.3-\mathrm{m} \mathrm{RC}$ & $50 / 70 \mathrm{~cm}$ \\
\hline CCD type: & AT200 & CH360 & ST-8 \\
Chip: & SITe SI003AB & SITe SI003B & KAF 1602E \\
Size: & $1024 \times 1024$ & $1024 \times 1024$ & $1530 \times 1020$ \\
Pixel size: & $24 \times 24 \mu \mathrm{m}$ & $24 \times 24 \mu \mathrm{m}$ & $9 \times 9 \mu \mathrm{m}$ \\
Scale: & $0.33^{\prime \prime} /$ pixel & $0.5^{\prime \prime} / \mathrm{pixel}$ & $1.1^{\prime \prime} / \mathrm{pixel}$ \\
Field: & $5.6^{\prime} \times 5.6^{\prime}$ & $8.5^{\prime} \times 8.5^{\prime}$ & $28^{\prime} \times 18.7^{\prime}$ \\
Gain: & $4.93 \mathrm{e}^{-} / \mathrm{ADU}$ & $5.3 \mathrm{e}^{-} / \mathrm{ADU}$ & $2.3 \mathrm{e}^{-} / \mathrm{ADU}$ \\
RON: & $3.9 \mathrm{ADU} / \mathrm{rms}$ & $2.6 \mathrm{ADU} / \mathrm{rms}$ & $6.2 \mathrm{ADU} / \mathrm{rms}$ \\
\hline
\end{tabular}

profile of the $\mathrm{H} \alpha$ line was seen. Detailed spectral investigation of CB34V was made by Alves et al. (1997). These authors report results from intermediate and high resolution spectral observations. They determined the spectral class as G5 (III-IV), the mass as $2 M_{\odot}$ and the age as $10^{6}$ years. A weak two component $\mathrm{H} \alpha$ emission line was found in the high resolution spectrum. The two possible explanations of observed the outburst are: (1) a high accretion episode (FUOR) and (2) time variable extinction from non-uniform circumstellar environment and are discussed in detail. In spite of existing discrepancies, CB34V was included in some lists of FUOR objects (Sandell \& Weitraub 2001). According to classification in GCVS, CB34V was named V 1184 Tau (Kazarovets et al. 2000).

In this paper we report data from optical photometry and spectroscopy of V 1184 Tau. Our investigation has as an objective a clarification of the nature of this object. We try to use accumulated data to determine the type of variability.

\section{Observations}

Our photometric and spectroscopic data were performed in two observatories with tree telescopes: the 2-m Ritchey-CretienCoude and 50/70/172 cm Schmidt telescopes of the National Astronomical Observatory Rozhen (Bulgaria) and the 1.3-m Ritchey-Cretien telescope of the Skinakas Observatory ${ }^{1}$ of the Institute of Astronomy, University of Crete (Greece).

\subsection{Photometry}

The photometric $U B V R I$ data reported in this paper was collected from October 2000-April 2002. Photometrics CCD cameras with the 2-m RCC and 1.3-m RC telescopes and SBIG ST8 camera with the $50 / 70 \mathrm{~cm}$ Schmidt telescope were used. The technical parameters and chip specifications for the cameras used are summarized in Table 1. The typical exposure times are 60-120 s for $R$ and $I, 120-180 \mathrm{~s}$ for $V, 300 \mathrm{~s}$ for $B$ and 300-600 s for $U$ filter. All frames were taken through a standard Johnson-Cousins set of filters. Twilight flat fields in each filter were obtained each night. All frames obtained with the Photometrics cameras are bias subtracted and flat fielded. CCD frames obtained with the ST8 camera are dark subtracted

\footnotetext{
${ }^{1}$ Skinakas Observatory is a collaborative project of the University of Crete, the Foundation for Research and Technology - Hellas, and the Max-Planck-Institut für Extraterrestrishe Physik.
}

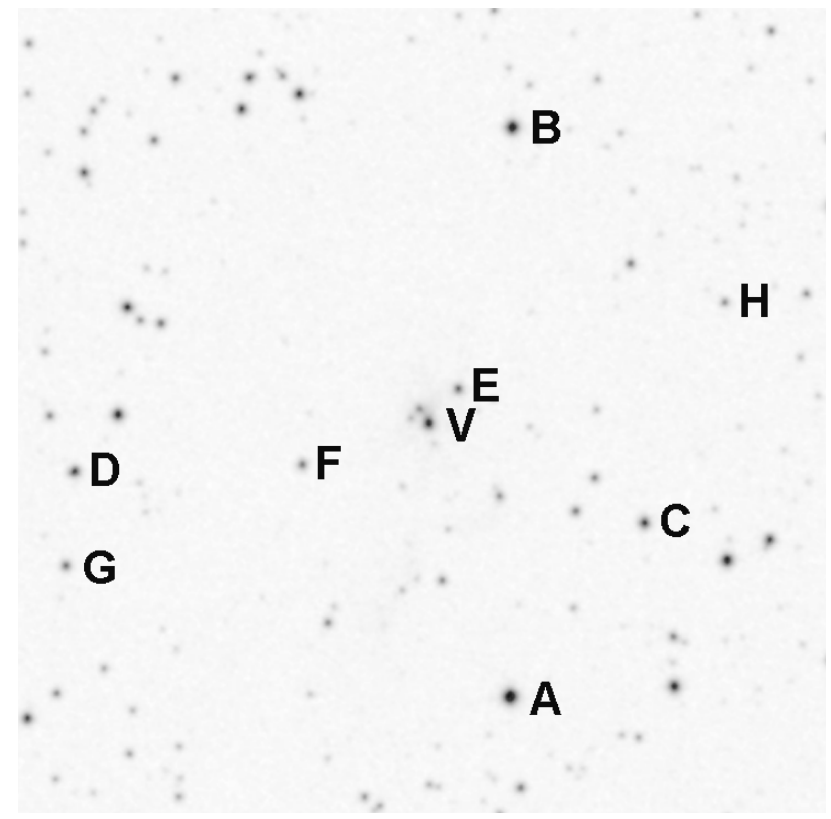

Fig. 1. A finding chart for the UBVRI comparison sequence around V 1184 Tau.

and flat fielded. Aperture photometry was performed using DAOPHOT routines.

In order to facilitate transformation from instrumental measurements to the standard system a sequence of eight comparison stars in the field of V 1184 Tau was calibrated in UBVRI bands. Calibration was made during five clear nights, three with the $1.3-\mathrm{m} \mathrm{RC}$ telescope and two with the $50 / 70 \mathrm{~cm}$ Schmidt telescope. Standard stars from Landolt (1992) were used as a reference. At least six standard fields in different air masses were observed every night. Table 2 contains the measured $U B V R I$ magnitudes and corresponding mean errors of the mean for comparison sequence. The stars are labeled from A to $\mathrm{H}$ in order of their $V$-band magnitude. The standards range from $V=13$. 264 to $V=17$ m. 005 and from $B-V=0$. 640 to $B-V=2$. 131 . The finding chart of the comparison sequence is presented in Fig. 1. The field is $6^{\prime} \times 6^{\prime}$, centered on V 1184 Tau. North is at the top and east to the left. The chart is retrieved from the STScI Digitized Sky Survey Second Generation Red.

The results of our photometric observations are summarized in Table 3. The columns give: date of observation, Julian date, $V$ magnitude, $U-B, B-V, V-R$ and $V-I$ color indices, telescope and CCD camera used.

\subsection{Spectroscopy}

Two long-slit optical spectra of V 1184 Tau have been obtained with the 2-m RCC telescope of the Rozhen Observatory and with the $1.3-\mathrm{m} \mathrm{RC}$ telescope of the Skinakas Observatory. In order to investigate the most prominent spectral futures for PMS stars the red spectral range (5500-7200 $⿱$ ) was observed.

Observations in Rozhen were made on 24 March 2001 with the Photometrics AT200 CCD camera and a focal reducer attached to the RC focus of the telescope. A grating prism (300 lines per $\mathrm{mm}$ ) and $130 \mu \mathrm{m}$ slit were used. This 
Table 2. Photometric data for UBVRI comparison sequence.

\begin{tabular}{lllllllllll}
\hline \hline Star & $V$ & $\sigma_{V}$ & $R$ & $\sigma_{R}$ & $I$ & $\sigma_{I}$ & $B$ & $\sigma_{B}$ & $U$ & $\sigma_{U}$ \\
\hline $\mathrm{A}$ & 13.26 & 0.02 & 12.88 & 0.01 & 12.49 & 0.02 & 13.90 & 0.04 & 13.88 & 0.09 \\
$\mathrm{~B}$ & 14.67 & 0.03 & 13.31 & 0.05 & 11.94 & 0.03 & 16.80 & 0.10 & - & - \\
$\mathrm{C}$ & 15.03 & 0.02 & 14.55 & 0.01 & 14.10 & 0.03 & 15.80 & 0.04 & 15.95 & 0.11 \\
$\mathrm{D}$ & 15.27 & 0.02 & 14.75 & 0.02 & 14.29 & 0.04 & 16.15 & 0.05 & 16.53 & 0.09 \\
$\mathrm{E}$ & 15.88 & 0.03 & 15.39 & 0.02 & 14.91 & 0.03 & 16.72 & 0.05 & 16.84 & 0.10 \\
$\mathrm{~F}$ & 16.38 & 0.02 & 15.56 & 0.02 & 14.87 & 0.05 & 17.65 & 0.03 & 18.55 & 0.15 \\
$\mathrm{G}$ & 16.49 & 0.03 & 15.45 & 0.04 & 13.39 & 0.06 & 18.16 & 0.09 & - & - \\
$\mathrm{H}$ & 17.01 & 0.06 & 16.38 & 0.04 & 15.83 & 0.03 & 18.00 & 0.10 & - & - \\
\hline
\end{tabular}

Table 3. UBVRI photometric observations of V 1184 Tau.

\begin{tabular}{lllllllll}
\hline \hline Date & JD & $V$ & $U-B$ & $B-V$ & $V-R$ & $V-I$ & Tel & CCD \\
\hline 2000 Oct. 29 & 2451847.552 & 15.28 & - & 1.59 & 1.08 & 2.38 & S cm & ST8 \\
2000 Oct. 30 & 2451848.522 & 15.51 & - & 1.67 & 1.09 & 2.41 & S cm & ST8 \\
2000 Dec. 24 & 2451903.467 & 15.17 & - & 1.67 & 1.08 & 2.33 & S cm & ST8 \\
2001 Jan. 20 & 2451930.447 & 15.07 & - & 1.58 & 1.04 & 2.26 & S cm & ST8 \\
2001 Feb. 16 & 2451957.368 & 15.24 & - & 1.59 & 1.06 & 2.26 & S cm & ST8 \\
2001 Feb. 17 & 2451958.360 & 15.16 & - & 1.57 & 1.05 & 2.27 & S cm & ST8 \\
2001 Mar. 24 & 2451993.320 & 15.12 & - & 1.50 & 1.04 & 2.18 & $2 \mathrm{~m}$ & Phot \\
2001 Sep. 1 & 2452153.615 & 15.09 & - & - & - & 2.25 & $1.3 \mathrm{~m}$ & Phot \\
2001 Sep. 2 & 2452154.622 & 15.30 & 1.01 & 1.59 & 1.09 & 2.30 & $1.3 \mathrm{~m}$ & Phot \\
2001 Sep. 3 & 2452155.594 & 15.27 & 0.80 & 1.59 & 1.07 & 2.26 & $1.3 \mathrm{~m}$ & Phot \\
2001 Sep. 4 & 2452156.600 & 15.54 & - & 1.63 & 1.13 & 2.33 & $1.3 \mathrm{~m}$ & Phot \\
2001 Sep. 5 & 2452157.617 & 15.52 & - & - & - & 2.33 & $1.3 \mathrm{~m}$ & Phot \\
2001 Sep. 16 & 2452168.615 & 15.41 & 1.05 & 1.57 & 1.11 & 2.31 & $1.3 \mathrm{~m}$ & Phot \\
2001 Sep. 17 & 2452169.607 & 15.62 & 1.18 & 1.55 & 1.12 & 2.35 & $1.3 \mathrm{~m}$ & Phot \\
2002 Feb. 6 & 2452312.324 & 15.03 & - & 1.67 & 1.06 & 2.29 & $\mathrm{~S} \mathrm{~cm}$ & ST8 \\
2002 Feb. 7 & 2452313.340 & 15.20 & 0.92 & 1.70 & 1.04 & 2.34 & $\mathrm{~S} \mathrm{~cm}$ & ST8 \\
2002 Mar. 6 & 2452340.298 & 15.50 & - & - & 1.12 & 2.31 & $2 \mathrm{~m}$ & Phot \\
2002 Mar. 17 & 2452351.283 & 15.24 & - & 1.65 & 1.05 & 2.34 & $\mathrm{~S} \mathrm{~cm}$ & ST8 \\
2002 Mar. 18 & 2452352.334 & 15.25 & 0.97 & 1.60 & 1.04 & 2.33 & S cm & ST8 \\
2002 Apr. 8 & 2452373.269 & 15.36 & - & 1.55 & 1.04 & 2.29 & S cm & ST8 \\
2002 Apr. 10 & 2452375.304 & 15.03 & - & - & 1.00 & 2.20 & S cm & ST8 \\
\hline
\end{tabular}

combination yields a resolving power $\lambda / \Delta \lambda=250$. Two $1200 \mathrm{~s}$ exposure frames of the object were obtained and combined to form the final spectrum. An exposure of a $\mathrm{Ne}-\mathrm{Ar}$ comparison lamp obtained after the finish of observations was used for a wavelength calibration. Standard star spectra were not obtained for flux calibration.

Observations in Skinakas were carried out on 9 September 2001 with ISA 612 spectral CCD camera $(2000 \times 800$ pixels, $15 \times 15 \mu \mathrm{m})$ and a focal reducer. A grating prism (1300 lines per $\mathrm{mm}$ ) and $160 \mu \mathrm{m}$ slit were used. This combination yields a resolving power $\lambda / \Delta \lambda=1300$. Two $1200 \mathrm{~s}$ exposures of V 1184 Tau were obtained followed immediately by an exposure of a $\mathrm{Ne}-\mathrm{Ar}$ comparison lamp.

The spectral data were reduced using a similar procedure with IDL routines. All frames were bias subtracted, flat fielded and cleaned of cosmic rays. A one-dimensional spectrum of the source was extracted using the command "total". For the sky subtraction we used two pairs of rows at each side of the spectrum. The spectrogram obtained with the 2-m RCC telescope is presented in Fig. 2. From the spectra obtained with the 1.3-m RC telescope we present the line profiles of $\mathrm{H} \alpha$, [OI] and Na I doublet (Fig. 3).

\section{Results and discussion}

Photometric variability is the most prominent property of PMS stars. Since there is only one photometric measurement of V 1184 Tau (Yun et al. 1997) after the outburst it is of great interest to study its variability at the present time. The $V$-light curve of V 1184 Tau in the period of our observations is shown in Fig. 4. It is seen from the figure that the star brightness varies with an amplitude of about $0 . \mathrm{m} 6$ without a trend toward increasing or decreasing. Such brightness variations can be seen in the other passbands. Our photometric data confirm the observation 


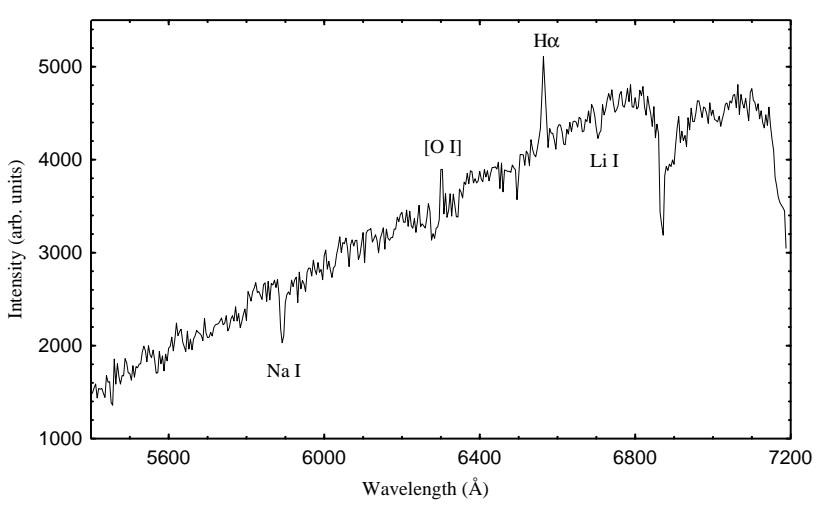

Fig. 2. Optical spectrum of V 1184 Tau obtained with the 2-m RCC telescope in Rozhen Observatory.
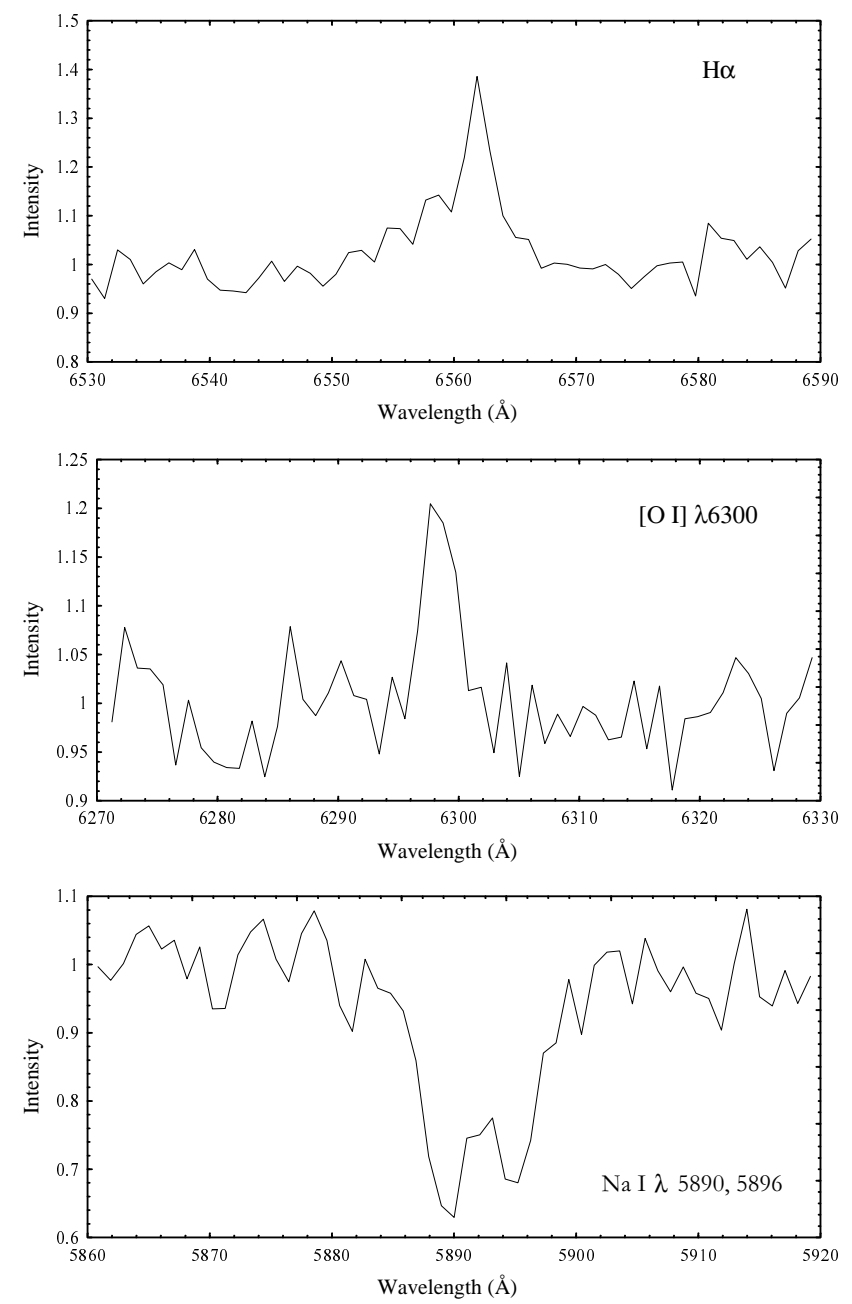

Fig. 3. Profiles of $\mathrm{H} \alpha,[\mathrm{OI}]$ and $\mathrm{Na}$ I lines obtained with the 1.3-m $\mathrm{RC}$ telescope in Skinakas Observatory.

by Yun et al. (1997) of near infrared excess emission. On the other hand there is no photometric evidence for the presence of ultraviolet excess. The measured $U-B$ color indices fit with the G5 (III-IV) spectral type determined by Alvas et al. (1997). The BVRI magnitudes of V 1184 Tau in 1996 estimated by Yun et al. (1997) have values close to our magnitudes in the period

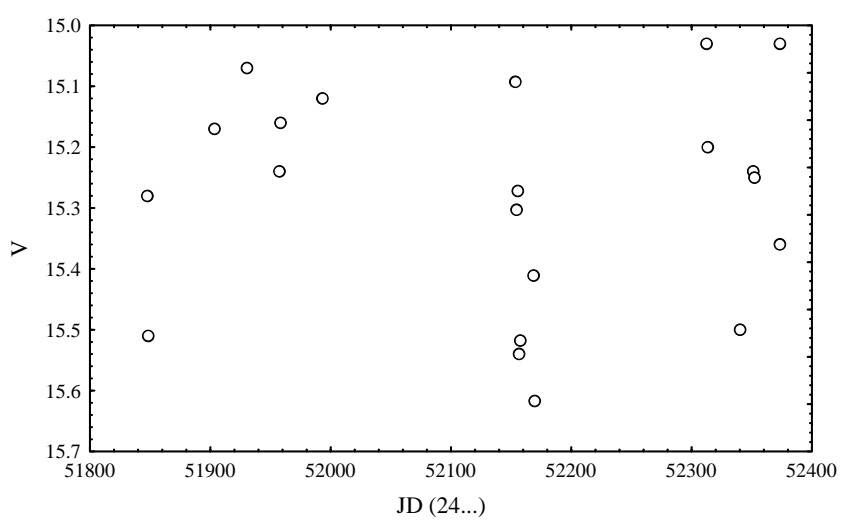

Fig. 4. A $V$-light curve of V 1184 Tau in the period October 2000-April 2002.

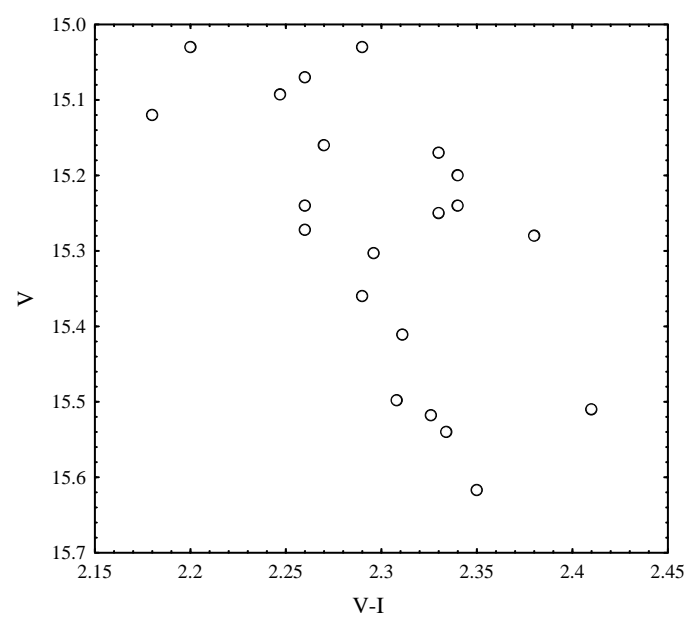

Fig. 5. Relationship between $V$ magnitude and $V-I$ color index for the period of observations.

of observations. Therefore, V 1184 Tau has kept its maximum brightness at least for the past 6 years.

Another interesting result from our photometric study is the variation of color indices with stellar brightness. In Fig. 5 we plot the measured color index $V-I$ versus stellar magnitude $V$ during the period of our observations. A clear dependence can be seen from the figure: the star becomes redder as it fades. Such color variations are typical of stars with large cool spots whose variability is produced by rotation of the spotted surface (WTTS). Most WTTSs show periodic variations with periods from 0.5 to 18 days (Herbst et al. 1994). Currently our data are too limited to search for periodicity, but we will try to collect a long set of observations for future studies.

Comparison of our spectral observations with the spectra obtained in 1995 (Yun et al. 1997) and 1996 (Alves et al. 1997) reveals major changes in the $\mathrm{H} \alpha$ line profile. While the emission peak in the $\mathrm{H} \alpha$ line increased considerably in strength, the sodium doublet keeps its absorption strength and equivalent width. The equivalent width of the $\mathrm{H} \alpha$ line measured from Skinaka's spectrum is $4 \AA$. Another typical spectral feature of PMS stars - the forbidden [OI] ( $\lambda 6003)$ line - is also seen in emission in our spectra. The peaks of the two emission lines are blueshifted by about $180 \mathrm{~km} \mathrm{~s}^{-1}$. The $\mathrm{H} \alpha$ emission line profile is not symmetric and the presence of a second blueshifted peak 
is suspected. According to Alves et al. (1997), V 1184 Tau is a rapidly rotating star which increases the difficulty in detecting other spectral features. None of the identified lines was found to exhibit a P Cygni profile in our spectra.

Line profile variability is a common property of PMS stars. This is especially true for the $\mathrm{H} \alpha$ emission line - the most prominent feature in the TTSs spectra. The recent study of BBW76 made by Reipurth et al. (2002) shows that significant profile variability of the emission and absorption features can be observed also in the spectrum of FUOR type objects.

Thus, our photometric and spectroscopic results suggest that V 1184 Tau has the characteristics of a WTTS. The observed variability in optical wavelengths (Type I from the Herbst et al. (1994) classification), the presence of $\mathrm{H} \alpha$ and $[\mathrm{OI}]$ in emission and $\mathrm{Li}$ in absorption, the equivalent width of $\mathrm{H} \alpha(4 \AA)$, the presence of an infrared excess and the absence of an ultraviolet excess (typical for CTTSs) strongly support this statement. On the other hand the observed optical outburst followed by minor activity around the maximum brightness resembles the light-curves of well-studied FUORs. This discrepancy makes the exact classification of V 1184 Tau difficult.

Acknowledgements. The author thanks the Director of Skinakas Observatory Prof. I. Papamastorakis and Dr. I. Papadakis for the telescope time.

\section{References}

Alves, J. F., Hartman, L., Briceno, C., \& Lada, C. J. 1996, AJ, 113, 1395

Alves, J. F., \& Yun, J. L. 1995, ApJ, 438, L107

Appenzeller, I., \& Mundt, R. 1989, A\&ARv, 1, 291

Bastian, U., Finkenzeller, U., Jaschek, C., \& Jaschek, M. 1983, A\&A, 126,438

Bertout, C. 1989, ARA\&A, 27, 351

Clemens, D. P., \& Barvainis, R. E. 1988, ApJS, 68, 257

Hartmann, L., \& Kenyon, S. J. 1996, ARA\&A, 34, 207

Herbig, G. H. 1962, Adv. Astron. Astrophys., 1, 47

Herbig, G. H. 1977, ApJ, 217, 693

Herbst, W., Herbst, D. K., \& Grossman, E. J. 1994, AJ, 108, 1906

Joy, A. H. 1945, ApJ, 102, 168

Kazarovets, E. V., Samus, N. N., \& Durlevich, O. V. 2000, IBVS, No. 4870

Khanzadyan, T., Smith, M. D., Gredel, R., Stanke, T., \& Davis, C. J. 2002, A\&A, 383, 502

Landolt, A. U. 1992, AJ, 104, 340

Martin, E. L. 1997, A\&A, 321, 492

Reipurth, B., Hartmann, L., Kenyon, S. J., Smette, A., \& Bouchet, P. 2002, AJ, 124, 2194

Sandell, G., \& Weintraub, D. A. 2001, ApJS, 134, 115

Yun, J. L., Moreira, M. C., Alves, J. F., \& Storm, J. 1997, A\&A, 320, 167 